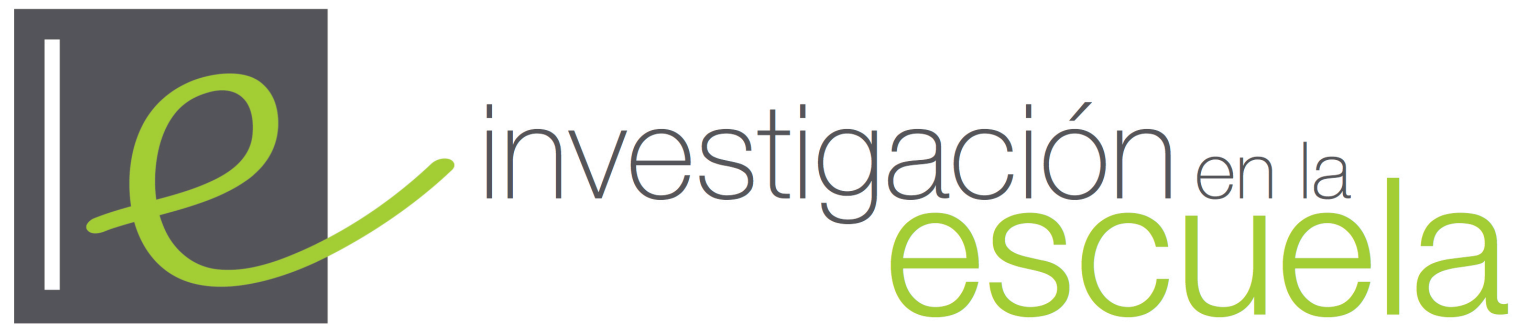

Revista internacional de investigación e innovación educativa

\title{
El currículum oculto del fracaso escolar y del abandono educativo temprano. Razones interseccionadas
}

\author{
Rosa Várquez Recio, Mónica López-Gily Guadalupe Calvo-García \\ Universidad de Cádiz \\ España
}

Citación: Vázquez Recio, R., López-Gil, M. y Calvo-García, G. (2019). El currículum oculto del fracaso escolar y del abandono educativo temprano. Razones interseccionadas. Investigación en la Escuela, 98, 16-30. doi: http://dx.doi.org/10.12795/IE.2019.198.02

Resumen: El presente trabajo es resultado de la investigación "Riesgo de fracaso y abandono escolar en Secundaria Obligatoria. Los contextos educativos, familiares y socioculturales. Un estudio cualitativo" (P12-SEJ-2664). Partiendo de los datos disponibles sobre el fracaso y el abandono escolar, y recurriendo a una perspectiva interseccional, que permite visibilizar y comprender el entramado de variables y sistemas que intervienen, se realiza un análisis de lo que denominamos el "currículum oculto" del fracaso y del abandono. Dicho análisis se fundamenta en las voces de adolescentes y jóvenes. Se ha adoptado un marco de investigación cualitativo y se ha utilizado el Software Nvivo para el tratamiento de los datos. Del análisis se desprende la necesidad de atender a esos componentes, actitudes, valores, etc., pertenecientes a contextos diversos (familiar, educativo, social) y sistemas diferentes (económico, político, cultural, patriarcal...) desde la interseccionalidad. Palabras clave: "Fracaso escolar"; "abandono temprano"; "interseccionalidad"; "educación secundaria"; "profesorado"; "currículum oculto"; "desenganche”; "investigación cualitativa". 
The hidden curriculum of school failure and early educational dropout. Intersected Reasons Abstract: This paper is the result of the research "Risk of failure and drop out out in obligatory secondary school. Educational, family and sociocultural contexts. A qualitative study "(P12-SEJ2664). Based on the available data on school failure and drop out, and using an intersectional perspective, which allows visualize and understand the structure of variables and systems involved, an analysis is made of what we call the "hidden curriculum" of school failure and drop out. This analysis is based on the voices of adolescents and young people. A qualitative research framework has been adopted and the Nvivo Software has been used for data processing. The analysis shows the need to attend to these components, attitudes, values, etc., belonging to diverse contexts (family, educational, social) and different systems (economic, political, cultural, patriarchal ...) from the intersectionality.

Key words: "School failure"; "dropo ut"; "intersectionality"; "secondary school”; "teacher"; "hidden curriculum"; "unhook"; "research qualitative".

Le programme caché de l'échec scolaire et de l'abandon scolaire précoce. Raisons croisées Resumè: Notre travail est le résultat de la recherche « Riesgo de fracaso y abandono escolar en Secudnaria Obligatoria. Los contextos educativo, familiares y socioculturales. Un estudio cualitativo (P12-SEJ-2664). À partir des données relatives à l'échec et le décrochage scolaire, et depuis une perspective intersectionnelle, qui nous permettre de montrer et comprendre le réseau de facteurs et dispositifs liés à ces phénomènes, nous développons une analyse de cela que nous appelons le « curriculum caché » de l'échec et le décrochage scolaire. Cette analyse est soutenue par les témoignages d'adolescents et jeunes. Nous avons choisi une méthodologie qualitative et on a utilisé le software Nvivo pour la gestion des informations. Ce travail nous montre la nécessité de porter l'attention sur les habitus, attitudes, valoir, etc. liés aux contextes (familial, éducatif, social) et aux dispositifs (économique, politique, culturel, patriarcale) à partir d'une regard intersectionnelle. Mots-clés: "Échec scolaire"; "décrochage scolaire"; "intersectionnalité"; "études du second degré"; "enseignants"; "adolescents"; "curriculum cache"; "recherche qualitative".

\section{Introducción}

El trabajo se fundamenta en la investigación "Riesgo de fracaso y abandono escolar en Secundaria Obligatoria. Los contextos educativos, familiares y socioculturales. Un estudio cualitativo" (Consejería de Innovación, Ciencia y Empresa Junta de Andalucía, P12-SEJ-2664). Partiendo de los datos disponibles sobre el fracaso y el abandono escolar, y recurriendo a una perspectiva interseccional, que permite comprender el entramado de variables y sistemas que intervienen, se realiza un análisis de lo que denominamos el "currículum oculto" del fracaso y del abandono, y lo hacemos desde las voces de adolescentes y jóvenes participantes en la investigación. Esta mirada metafórica nos sitúa en ese lado de los fenómenos que se halla configurado por una serie de elementos, valores, actitudes, etc., que generalmente no son nombrados en la concurrencia directa del fracaso y del abandono. Hablar de las metodologías docentes, de las formas organizativas de las aulas y de los centros, de la disciplina y del orden, del libro de texto como recurso central y exclusivo, etc., supone situarnos en ese currículum oculto.

En el presente trabajo abordaremos el estado de la cuestión del fracaso y del abandono, ofreciendo una visión general, para pasar, desde esta a la dimensión interseccional. La interseccionalidad se presenta como una herramienta de análisis crítico y político de ambos fenómenos que evidencia claramente la complejidad de estos. Este proceso de análisis nos permite concretar el 
currículum oculto, aportando aspectos silenciados o tratados con menor relevancia en la concurrencia del fracaso y del abandono.

\section{La oficialidad del fracaso escolar y el abandono temprano: lo tangible}

El fracaso escolar y el abandono educativo temprano constituyen asuntos de especial relevancia en el contexto español, pero también a nivel internacional, como lo evidencian diferentes informes (Serrano, Soler y Hernández, 2013; Eurostat, 2019; Ministerio de Educación, Cultura y Deporte, 2017a, 2017b; OECD, 2017; Schraad-Tischler, Schiller, Matthias Heller \& Siemer, 2017; OREAL/Unesco, 2013; Suárez, 2018; Ministerio de Educación y Formación Profesional, 2018, 2019), así como la amplia literatura producida (Rumberger, 2001; Marchesi, 2003; Escudero Muñoz, 2005; Ferrer Esteban et al., 2006; Rumberger y Ah Lin, 2008; Bolívar y López, 2009; Alegre y Benito, 2010; Calero, Choi y Waisgrais, 2010; Witte et al., 2014; Estrada de Madariaga, 2017). Con ello se intenta contribuir a la explicación de ambos fenómenos para diseñar e implementar medidas que den respuesta a tales situaciones. En términos generales, y tomando la definición que establece la Comisión Europea con el propósito de que sea común para todos los países de la Unión Europea (UE), el abandono educativo (school leavers, empleando la denominación europea), remite a jóvenes de 18 a 24 años que no están escolarizados y que no han completado la educación secundaria obligatoria o anteriores niveles educativos. Atendiendo a la nomenclatura fijada para este fenómeno, se trata de estudiantes que posee únicamente ISCED $^{1}$ 0, 1, 2, y 3 (UNESCO, 2012). Por otra parte, para el fracaso escolar disponemos de definiciones diversas que reflejan en sí su complejidad. Como señala Martínez García (2009, p. 56), "el fracaso escolar es una expresión que tiene la virtud de señalar de forma inmediata una realidad social, pero el defecto de cargar dicha realidad negativamente", consideración que pone en valor la reflexión de Carabaña (2009, p. 76): “el fracaso escolar es un arbitrario administrativo que no refleja el aprendizaje de los alumnos y carece de validez y de frabilidad como indicador de la calidad de la enseñanza. Más aún, en la medida en que tiene consecuencias sociales, su arbitrariedad es una grave injusticia". Asumiendo lo que conlleva el constructo, y atendiendo a la literatura, por fracaso escolar podemos entender aquel alumnado que no obtiene el título educativo obligatorio y aquellos que no alcanzan el nivel de conocimientos mínimos ni el esperado rendimiento académico fijado para la educación obligatoria. Para dar un sentido más contextualizado, dinámico e interactivo, es preciso considerar el concepto "situación de riesgo" (at risk), en tanto que remite a esa situación a la que se encuentra expuesto el estudiantado la cual se caracteriza por los factores de riesgo y/o las condiciones particulares que hace que este tenga más probabilidad de experimentar consecuencias adversas o desfavorables (Finn \& Rock, 1997, pp. 221-222).

Salvando las diferencias entre países de la UE, ambos fenómenos vienen a ser una constante, si bien se aprecia una distribución un tanto heterogénea entre países, del 3,1\% que presenta Croacia hasta el 18,6\% de Malta (Eurostat, 2019), situándose España con un porcentaje de 17,9\% en el 2018, la tasa más baja alcanzada (Ministerio de Educación y Formación Profesional, 2019). En el intervalo de diez años (2008-2018) se ha reducido 13,8 puntos.

El objetivo europeo marca una tasa del 10\%, tal y como queda recogido en la Estrategia 2020, Europa 2020: Una estrategia para el crecimiento inteligente (Comisión Europea, 2010); España se ha comprometido cumplir con los objetivos marcados por la Comisión Europea, fijando para el año 2020 una tasa de abandono por debajo del 15\%. Este interés queda expresado a través de los

\footnotetext{
${ }^{1}$ ISCED: International Standard Classification of Education. El nivel 0 corresponde a la educación infantil; el nivel 1, a la educación primaria; el nivel 2, a la secundaria inferior (primer ciclo de la ESO en España), y el nivel 3, a la secundaria superior (segundo ciclo de la ESO en España) (UNESCO, 2012).
} 
Programas Nacionales de Reforma (PNR), y en concreto en el Programa Nacional de Reformas de España de 2018 (Ministerio de Empleo y Seguridad Social, 2018). Las actuaciones contempladas para minimizar la tasa de abandono se enmarcan en el Plan para la reducción del abandono educativo temprano (Ministerio de Educación, Cultura y Deporte, 2015). Las líneas de actuación son prevención, intervención y compensación: "la prevención para evitar desde el principio las condiciones que pueden desencadenarlo, la intervención para abordar las nuevas dificultades en una fase temprana y las medidas compensatorias que ofrecen oportunidades de educación y formación a quienes han abandonado el sistema educativo" (2015, p.11). En el año 2017 fue aprobado el Programa de cooperación territorial para el avance y apoyo en la educación y permanencia en el sistema educativo, "PROEDUCAR", para reducir el abandono temprano de la educación y la formación a través de mecanismos de apoyo (Ministerio de Empleo y Seguridad Social, 2018, p. 34). Asimismo, mencionar el Programa Operativo de Empleo, Formación y Educación (POEFE), que tiene como una de sus prioridades establecer actuaciones para la reducción y prevención del abandono escolar temprano (Ministerio de Empleo y Seguridad Social, 2018, p. 56).

Si atendemos a las Comunidades Autónomas también encontramos diferencias. Sin entrar en un análisis pormenorizado que pueda explicar las diferencias entre los valores alcanzados por cada comunidad autónoma, en el año 2018 la tasa más elevada la presenta Melilla con el 29, 5\%, seguida de Islas Baleares (24,4\%) y Andalucía (21, 9\%), mientras que Cantabria alcanza el 9,8\% (tabla 1).

Tabla 1

Abandono temprano por comunidad. Año 2018.

\begin{tabular}{|l|r|}
\hline Comunidad Autónoma & $\mathbf{2 0 1 8}$ \\
\hline Andalucía & 21,9 \\
\hline Aragón & 15,8 \\
\hline Asturias, Principado de & 12,6 \\
\hline Balears, Illes & 24,4 \\
\hline Canarias & 20,9 \\
\hline Cantabria (3) & 9,8 \\
\hline Castilla y León & 13,9 \\
\hline Castilla-La Mancha & 20,5 \\
\hline Cataluña & 17,0 \\
\hline Comunidad Valenciana & 20,2 \\
\hline Extremadura & 20,9 \\
\hline Galicia & 14,3 \\
\hline Madrid, Comunidad de & 14,4 \\
\hline Murcia, Región de & 24,1 \\
\hline Navarra, Comunidad Foral de & 11,4 \\
\hline País Vasco & 6,9 \\
\hline La Rioja & 17,1 \\
\hline Ceuta & 23,4 \\
\hline Melilla & 29, \\
\hline TOTAL & $\mathbf{1 7 , 9}$ \\
\hline Nota: Fuente: Ministerio & $\mathrm{ed}$ \\
\hline
\end{tabular}

Nota: Fuente: Ministerio de Educación y Formación Profesional, 2019.

Por otra parte, en relación a la población de 16-19 años titulada al menos en ESO o niveles similares, Cantabria tiene la tasa más elevada (87,45\%) frente a Ceuta y Melilla (69,6\%) (Ministerio 
de Educación y Formación Profesional, 2019); Andalucía presenta una tasa del 76,5\%. Con respecto a la población con titulación inferior a la segunda etapa de la Educación Secundaria, el 69,8\% se concentra en el tramo de edad de 16 a 19 años, y el 24,2\% entre los 20 y 24 años (tabla 2).

Tabla 2

Abandono educativo temprano por nivel de formación y edad

\begin{tabular}{cccc}
\hline Edad & Total & Con título de ESO & Sin título de ESO \\
\hline $\mathbf{1 8}$ años & 11,6 & 6,5 & 5,1 \\
\hline $\mathbf{1 9}$ años & 15,0 & $\mathbf{8 , 7}$ & $\mathbf{6 , 3}$ \\
$\mathbf{2 0}$ años & 17,5 & 10,7 & 6,8 \\
\hline $\mathbf{2 1}$ años & 19,3 & 12,3 & $\mathbf{7 , 0}$ \\
\hline $\mathbf{2 2}$ años & 19,7 & 12,6 & 7,1 \\
\hline $\mathbf{2 3}$ años & 20,6 & $\mathbf{1 2 , 5}$ & $\mathbf{8 , 1}$ \\
\hline $\mathbf{2 4}$ años & 22,1 & 13,4 & 8,7 \\
\hline
\end{tabular}

Nota: Fuente: Ministerio de Educación y Formación Profesional, 2019.

Paralelamente a este conjunto de datos que nos permite hacer una cartografía numérica del estado de la cuestión en nuestro país y en nuestra comunidad autónoma, contamos con una vasta literatura, tanto a nivel nacional como internacional, que adoptan perspectivas distintas (no siempre complementarias), al tiempo que toman como foco prioritario ciertas dimensiones y no otras, y desarrollan investigaciones con planteamientos metodológicos distintos. A modo de mapeo, el estudio de Ferrer Esteban, Castel Baldellou y Ferrán Ferrer (2006) revisa la persistencia de variables fundamentalmente relacionadas con los centros y sus características, y contemplan otras variables de no menor importancia como la autoconfianza y las aspiraciones del alumnado. Calero, Choi y Waisgrais (2010) toman variables personales, familiares y escolares que determinan de manera taxativa un alto grado el riesgo de fracaso escolar. Otros factores que se aportan tienen que ver con la inmigración (Calero, Escardíbul, Waisgrais y Mediavilla, 2007), con el origen social (Bernardi y Requena, 2010); Casquero y Navarro (2010) analizan el abandono atendiendo a la variable género, y el trabajo de Mora (2010) se centra en los determinantes del abandono escolar. Por su parte, Breen \& Jonsson (2005) señalan que el rendimiento académico está segmentado por clases sociales. Estas investigaciones van en la línea de las de Escudero Muñoz (2005), Marchesi (2000, 2003), Rumberger y Ah Lin (2008), Lizasoain, Joaristi, Lukas y Santiago (2007), Moriña Díez (2007) y Escudero Muñoz, González González y Martínez Domínguez (2009). Cabe decir al respecto que han predominado investigaciones que arrojan una información que evidencia cuantitativamente la relevancia estadística de los factores implicados en la concurrencia del fracaso y/o abandono, y la indudable presencia de estos con mayor o menor poder de determinación. No obstante, las evidencias cuantitativas necesitan forzosamente de estudios que nos sitúen de modo directo en los sujetos que terminan fracasando y/o abandonan el sistema, y ya contamos con trabajos como los de Gamoran (2001), Susinos Rada y Calvo Salvador (2005-2006), Susinos Rada y Parrilla Latas (2008), Mena Martínez, Fernández Enguita y Riviére Gómez (2010), Fernández Enguita, Mena Martínez y Riviére Gómez (2010), Parrilla Latas, Gallego Vega y Moriña Díez (2010), Calderón Almendros (2014, 2016a, 2016b) y Vázquez Recio (2018). 


\section{Los claroscuros del fracaso escolar y el abandono educativo}

El panorama dibujado a partir de los datos disponibles por los estudios señalados refleja un mapa complejo de variables y factores que aglutina, al mismo tiempo, las características del sistema educativo, de los centros educativos y las aulas, las particularidades de los contextos familiares y también las características del alumnado. Sin embargo, es la propia complejidad que deviene la que hace tomar en consideración no tanto, ni tan solo, el conjunto de variables y factores que pueden intervenir, sino la trama que resulta de la interacción entre los mismos. Su potencial, en definitiva, deriva del efecto de esa interacción e interdependencia que pueda darse, en tanto en cuanto determina las formas de desigualdad, discriminación y exclusión (o de "privilegio"). Esta perspectiva nos la ofrece la herramienta de análisis crítico y político denominado interseccionalidad (Hill Collins, 1999, 2009; Hill Collins y Bilge, 2016). Con ella es posible poner de manifiesto que tanto el fracaso como el abandono no dejan de ser constructos con una carga ideológica que conecta, precisamente, con las políticas sociales, educativas y económicas que generan injusticias y desigualdad. Este posicionamiento permite contrarrestar las prácticas despolitizadoras que promueve el neoliberalismo y que hacen responsables a los sujetos de sus éxitos y sobre todo de sus fracasos. La meritocracia que se promueve y a la que tienen que aspirar toda la población adolescente y joven no deja de ser una falsa meritocracia, dado que de partida no todos disfrutan de igualdad de oportunidad y de derechos. Asumir la interseccionalidad ayuda a que no se naturalice la desigualdad, la inequidad y la injusticia que viene propiciada por la violencia estructural que funge las políticas neoliberales y neoconservadoras hermanadas con el capitalismo tecnocrático de nuestros días.

A tenor de lo expuesto, para comprender el fracaso y el abandono escolar resulta fundamental conocer cómo dichas políticas conforman nuestra sociedad actual. No podemos perder de vista que vivimos en la sociedad de la información, en la que lo más importante es la recopilación de datos acerca de los hábitos de la ciudadanía, a la que se le ofrecen los discursos que benefician a quienes ostentan el poder, las grandes empresas multinacionales; que dichas empresas están sustentadas por la economía de mercado propia de la ideología neoliberal dominante, que les otorgan todo tipo de privilegios y les permiten moverse por el mundo globalizado utilizando a su antojo los recursos naturales y a las personas.

En este contexto surgen los nuevos pobladores de la escuela a los que alude Jorganes (2007). Entre ellos están los niños y las niñas excluidos por la precariedad laboral, cuyos padres viven en la continua inestabilidad económica y emocional, o están ausentes porque han partido a trabajar a otros lugares, o los han llevado a otros en busca de una oportunidad. Estos niños y estas niñas son también los nuevos pobres de la sociedad consumista, rechazados en ocasiones por no llevar la ropa de moda o no tener el último móvil, pero igualmente por no poder pagar excursiones, materiales escolares o clases particulares que le permitan aprobar las diferentes asignaturas. Niños y niñas solos, porque sus familiares se ven obligados a cumplir las normas que impone el neoliberalismo asumiendo jornadas laborales interminables, y cuyas tardes son acompañadas de la televisión, los videojuegos o internet.

Este alumnado, víctima del neoliberalismo salvaje en sus contextos familiares, como adelantábamos, debe lidiar con el mismo, igualmente, cuando se convierte en "recursos" en un contexto escolar totalmente condicionado por el mercado. De manera especial, como señala Torres Santomé (2017), desde el año 2000, cuando la OCDE pone en marcha las pruebas de evaluación PISA, que centran su atención en las materias valoradas por las empresas: matemáticas, lengua y ciencias, se toman como las pruebas más fiables para hacer el diagnóstico de la calidad de los sistemas educativos. Dichas pruebas están determinando las políticas educativas y condicionando la labor del profesorado, y dando lugar, como bien explica Robinson (2012), a que estudiantes con 
increíbles talentos, piensen que no los tienen, que no valen, que no son buenos... y a que fracasen en la escuela, y en algunos casos, terminen abandonando.

En España, los mandatos neoliberales y conservadores en el ámbito de la educación adquieren su máxima expresión en la LOMCE; una ley pensada para el desarrollo económico, reduce la democracia escolar, adelanta la edad para cursar itinerarios académicos alternativos, favorece a la escuela concertada, revaloriza la religión y el amor a la patria, recurre a las evaluaciones externas para clasificar a los centros, etc.

Pero la preparación de la infancia para adaptarse a la nuestra sociedad competitiva e individualista no se limita a la escuela. Como bien relata Vázquez Recio (2015), si durante el tiempo escolar no se respetan sus ritmos, sus necesidades y sus motivaciones, en el tiempo extraescolar, tampoco. La jornada de los niños y las niñas se llena de actividades (incluidos los deberes) que persiguen prepararles para que sean competentes y emprendedores en el futuro, y no le dejan tiempo libre ni de ocio. Actividades que, además, deben acompañar o financiar unas familias que, como anticipábamos, no siempre cuentan con el tiempo, con la formación o con los recursos económicos necesarios. De este modo, va aumentado su desigualdad. Todo ello son factores que concurren de manera interseccional en la construcción del fracaso y del abandono.

\section{E1 mapeo metodológico: pretensiones y recogida de información}

La investigación desarrollada dentro del proyecto "Riesgo de fracaso y abandono escolar en Secundaria Obligatoria. Los contextos educativos, familiares y socioculturales. Un estudio cualitativo" (P12-SEJ-2664) ha sido de índole cualitativa. El acercamiento al estudio de las situaciones de riesgo al fracaso escolar y, por ende, al abandono temprano desde las percepciones y las experiencias de adolescentes y jóvenes requería de un soporte metodológico que permitiese ser coherente con las pretensiones de la investigación, y, al mismo tiempo, alcanzar un corpus empírico suficientemente sólido que garantizara credibilidad, confiabilidad y comprensibilidad (Flick, 2014). Investigar con un enfoque y compromiso cualitativo era la opción adecuada y pertinente para poder acceder al sentido y a los significados que adolescentes y jóvenes les atribuyen al fracaso y al abandono, conocer esa construcción que realizan desde las situaciones que los hacen ser sujetos pertenecientes a la categoría de "fracasados" y de "los que abandonan el sistema". También interesaba comprender cómo los contextos determinan el fracaso y/o abandono del alumnado (familiar, social y escolar).

Las estrategias empleadas han sido en concreto tres: entrevistas etnográficas, estudios de caso biográficos y grupos focales. Los sujetos informantes pertenecen al intervalo de edades comprendidas entre los 12 y los 26 años, y fueron seleccionados siguiendo dos criterios: a) estudiantes en riesgo, y b) abandono temprano ("early school leavers"). En relación a los grupos, nos ha interesado tanto el alumnado en riesgo y que han abandonado temprano el sistema, como aquellos que han retomado sus estudios cursando la Educación Secundaria para personas Adultas (ESPA).

Se han realizado un total de 91 entrevistas en las provincias implicadas en el estudio (Cádiz, Málaga y Almería), siendo un 33\% mujeres y un 67\% hombres. En relación a este aspecto, hay dos grandes grupos que señalar: el $46 \%$ de las personas entrevistadas tienen entre 12 y 16 años, grupo que tiene la obligación de permanecer en el sistema educativo, y el $54 \%$, con edades comprendidas entre 17 y 26 años que no tiene dicha obligación. Igualmente se han realizado ocho estudios de caso biográficos y ocho grupos focales. El análisis de los datos se ha realizado mediante la aplicación del software Nivo11, y ha permitido establecer una serie de nodos, siendo los de primer nivel el 
contexto familiar, el contexto escolar y la dimensión personal, y cada uno de ellos engloba una diversidad de elementos.

\section{Del claroscuro al currículum oculto del fracaso escolar y del abandono temprano}

Como antes destacábamos, el abordaje de las situaciones de riesgo al fracaso escolar y/o al abandono temprano, que un momento del proceso dejan de ser de riesgo para materializarse de facto, implica poner el foco de atención en la interseccionalidad, en tanto que define la compleja trama en la que tales fenómenos van tomando cuerpo con unas concreciones que varían en función de los sujetos portadores de los mismos. Dicha interseccionalidad lleva consigo la definición de una matriz de dominación, aspecto que resulta tremendamente útil para visibilizar aquellos aspectos interrelacionados que quedan en un segundo plano, o ni siquiera son considerados. Hill Collins (1999, p. 299) hace referencia a la matriz como "la organización general de las relaciones jerárquicas de poder en cualquier sociedad (...) Cualquier matriz particular de dominación se organiza a través de cuatro dominios de poder interrelacionados: estructurales, disciplinarios, hegemónicos e interpersonales". Estos cuatro dominios intervienen en la configuración del fracaso escolar y del abandono educativo y forman parte de lo que denominamos el currículum oculto de los mismos.

\section{El currículum oculto del fracaso y abandono}

Tradicionalmente el fracaso escolar y el abandono educativo han sido fenómenos cuyas causas son atribuidas al alumnado y su falta de interés hacia lo escolar, un nivel académico "de base" insuficiente, escasa habilidad para mantener la atención, etc. Sin embargo, el currículum, las prácticas docentes, el sistema educativo y su organización y sus exigencias no han formado parte del juicio sobre el fracaso y el abandono. Al respecto cabe preguntarse, ¿hasta qué punto el fracaso les pertenece únicamente a los llamados fracasados? Si bien el profesorado se atribuye parte del éxito escolar de un alumno o una alumna, ¿por qué no lo hace cuando se habla de fracaso? Lo mismo podemos platear en relación al sistema educativo. Sin duda, desde una mirada holística e interseccional, el currículum, el sistema educativo actual y la práctica docente deben ser cuestionadas como elementos que tienden a reproducir, pero también a producir las desigualdades y los dominios estructurales, disciplinarios e interpersonales que condicionan las vidas académicas y vitales del alumnado. Las personas que han fracasado en la escuela son aquellas que no responden a las expectativas de lo que el sistema entiende como alumnado ajustado al modelo esperado.

Sin embargo, los discursos de los chicos y las chicas informantes denuncian un currículum que, de forma sutil pero profunda, ahonda en el desenganche educativo y declaran elementos excluyentes y segregadores relacionados con el sistema escolar. El alumnado perteneciente a la categoría de "fracaso escolar" precisamente se sitúa en esa intersección de los diferentes factores.

Se deben analizar, por tanto, el currículum disciplinar, las dinámicas educativas y las relacionales que se producen en las aulas, los modelos organizativos burocráticos y jerárquicos, los contenidos presentes (y los ausentes: currículum nulo), metodologías y recursos se ofrecen y para qué alumnado está (o no) pensado. Desde esta mirada es posible dar un sentido a estos elementos, y son muchos los que de forma explícita, y otras veces oculta, van haciendo que en el proceso educativo se desplieguen actitudes de resistencia, desobediencia, desenganche, desafección, desdicha, desmotivación y otras tantas ausencias de actitudes y acciones favorables hacia la vinculación con lo escolar. Precisamente, el currículum oculto del fracaso y del abandono lo configura esa serie de 
elementos que no se ponen en la primera línea de análisis en tanto que tienen que ver con el orden curricular y organizativo hegemónico y homogenizador.

La recogida de datos evidencia la presencia de contenidos didácticos que distan mucho de lo relevante y significativo para el alumnado. Así se pone de manifiesto síntomas como el aburrimiento, la falta de interés, la desgana, la distracción con cualquier otro asunto que no sea aquello que se esté trabajando en clase. Unos contenidos que son percibidos como "inútiles", ajenos y desvinculados con su experiencia vital. La cultura popular no es la base sobre la que se construye la cultura académica, son dos mundos paralelos que viven (pero no conviven) en el espacio escolar. Los contenidos trabajados "son para otros", unos "otros" que hacen alusión a quienes sí se ajustan a los parámetros bajo los cuales se diseña el currículum oficial que actúa desde la óptica de la homogenización excluyente. Este asunto hace que el alumnado que no se siente parte de lo que ocurre en clase, cree mecanismos de defensa y resistencia (Willis, 1988) que les lleva a la búsqueda de actividades que les son más satisfactorias como escuchar música o charlar con compañeros o compañeras para aislarse emocional y cognitivamente de lo que ocurre en clase.

No hacía nada, no hacía tareas, ni nada, me sentaba en la silla y calentaba la silla como dicen, que me quedaba en el sitio y no hacía nada $\left(\mathrm{h} 17^{2}\right)$.

Solo explicaban a quienes sacaban buenas notas, si yo preguntaba, ni me miraba a la cara. (m17).

No me interesaba nada de lo que me contaban, la verdad. Me ponía hasta los cascos y con tal de que no molestara, me dejaban (h12).

El dominio hegemónico se pone en juego en el lenguaje que empleamos (accesible solo para unos pocos), la puesta en marcha de metodologías excluyentes y competitivas que exigen un papel pasivo del alumnado ante una cultura dominante neoliberal y neocolonialista que silencia, oculta o tergiversa las voces de ciertos colectivos sociales. Las metodologías de fuerte carácter academicista y uniformizadora inciden en la ausencia de significatividad de los contenidos. El conocimiento se ofrece de manera fragmentada y expositiva en clases en las que el profesorado, lejos de dialogar, "explica y explica casi sin respirar" (h16), mientras el alumnado oye, apunta y calla; un conocimiento que al alumnado le resulta aislado, ajeno y confuso y que no resuelve sus inquietudes y preocupaciones (y, en caso de hacerlo, solo las de unos pocos).

Es que parecen médicos explicando... hablan igual que ellos y yo no me entero de nada (m17).

Es que eso de explicar y explicar, al alumno le cansa. Las explicaciones eran interminables y terminaba todo reventado.

Las clases... nada, llega el maestro, nos habla y hacemos las tareas. (...) Claro el que habla es el profe y nosotros solo anotamos y hacemos actividades (h16).

Por otra parte, el libro de texto forma parte de este dominio. Es el único recurso didáctico utilizado e impone un ritmo, una cultura dominante y una forma de aprendizaje memorístico que solo favorece a quienes se acercan al modelo de estudiante en el que se ha basado su diseño. Llega a convertirse en la llave que permite entrar (o no) en la clase y a lo que se entiende como aprendizaje. A saber, quien no tiene libro de texto, ya sea por olvido o por graves dificultades económicas, puede

2 Los fragmentos de las entrevistas llevan un código (m/h/edad) que corresponde a la persona informante: " $\mathrm{m}$ " y " $\mathrm{h}$ ", se refieren a "mujer" y "hombre", y el número a la "edad". 
ser expulsado de clase y, por tanto, se le imposibilita acceder a la información que requiere para superar las pruebas con la que se evalúa.

Yo no podía comprar el libro y me decían que no podría aprender y a veces me echaba (h19). Tú llegabas sin libro y te decían venga pues $p a$ afuera, pal pasillo (h17).

A mí a veces ni me daba el examen para hacerlo, decía que para lo que iba a hacer... (m15).

De esta forma se interioriza una autoimagen negativa y baja confianza hacia el logro puesto que el fracaso llama al fracaso.

Si es que yo soy un caso perdido (h17).

Yo no sirvo para estudiar, es que no sirvo, soy muy torpe (m17).

El alumnado reclama dinámicas de clase basadas en el diálogo, la controversia, el debate, la argumentación y la reflexión que les ayude a desarrollar habilidades para desenvolverse en su contexto. Esta demanda requiere el uso de fuentes diversas, y no solo del libro de texto.

Así mismo resulta determinante respecto a la vinculación con lo educativo, las relaciones interpersonales que el alumnado mantiene con el profesorado, la confianza y las expectativas que este les tiene. La afectividad, el interés, la atención, la empatía, la confianza y las altas expectativas que el alumnado siente de su profesorado son directamente proporcional a su enganche escolar. El alumnado demanda reconocimiento a su persona, a sus logros, sus necesidades y dificultades; una atención personal que vaya más allá de lo estrictamente curricular y una mayor confianza en sus capacidades de superación. Son conscientes de que en secundaria las relaciones son diferentes, entendidas como débiles, jerárquicas, controladoras y curriculares, y basadas en el control y obtención de resultados académicos y no en el desarrollo de estrategias de desarrollo personal para afrontar las dificultades y presiones "extraescolares". Se pone en práctica el dominio interpersonal que incide en la construcción de identidades con baja autoestima.

Los maestros se creen que yo no sirvo para nada, y así ¿cómo lo hago? (m15)

Yo pregunto una duda, y no me hacen tanto caso como a otra persona que estudia más (h18).

El profesor daba su clase, y fuera..., no se preocupaba por nada ni nadie (h16).

Me sentía sola, no me veía con la capacidad; quiero tener un apoyo para que pueda sacar mis buenas notas y sacarme mi ESO (m17).

\section{Conclusiones}

Sin duda alguna, los discursos de los sujetos que sufren e incluso son víctimas de un currículum selectivo, basado en parámetros neoliberales y que de forma implícita les excluye y empuja al fracaso, son fuente rica de análisis de las prácticas docentes y del sistema educativo, de sus exigencias y sus dominios.

Estas voces, silenciadas y devaluadas por y en el currículum ayudan a reconocer el proceso que les ha llevado a desengancharse y, según casos, más que abandonar, asumir la expulsión del sistema educativo dominante. Se hace necesario, por tanto, el desarrollo de una formación y desarrollo profesional del docente desde la pedagogía crítica hacia la prevención e intervención del fracaso y abandono escolar, e igualmente adoptar una mirada que atienda a la trama compleja que define los sistemas y factores que inciden, con diferente grado y forma, en la concurrencia de las 
situaciones de riesgo al fracaso y al abandono. Nos sitúa en esa responsabilidad de ejercer como agentes sociales, intelectuales y comprometidos con una educación justa e inclusiva.

\section{Referencias}

Alegre, M. A., y Benito, R. (2010). Los factores del abandono educativo temprano. España en el marco europeo. Revista de Educación, núm. extr., 65-92.

Bernardi, F., y Requena, M. (2010). Inequality in educational transitions: the case of post-compulsory education in Spain. Revista de Educación, núm. extr., 93-118.

Bolívar, A., y López, L. (2009). Las grandes cifras del fracaso escolar y los riesgos de exclusión educativa. Profesorado. Revista de Currículum y Formación del Profesorado, 13(3). Disponible en: https://bit.ly/2DDpOga

Breen, R., \& Jonsson, J. O. (2005). Inequality of opportunity in comparative perspective: Recent research on educational attainment and social mobility. Annual Review of Sociology, 31, 223-243.

Calderón Almendros, I. (2014). Sin suerte pero guerrero hasta la muerte: pobreza y fracaso escolar en una historia de vida. Revista de Educación, 363, 184-209.

Calderón Almendros, I. (2016a). Fracaso escolar y desventaja sociocultural. Una aproximación biográfica. Barcelona: UOC.

Calderón Almendros, I. (2016b). Liberarse de la escuela. Historia de vida de Elena. Málaga: RIUMA.

Calero, J., Escardíbul, J. O., Waisgrais, S., y Mediavilla, M. (2007). Desigualdades socioeconómicas en el sistema educativo español. Madrid: Ministerio de Educación y Ciencia. Centro de Investigación y Documentación Educativa (CIDE).

Calero, J., Choi, A., y Waisgrais, S. (2010). Determinantes del riesgo de fracaso escolar en España: una aproximación a través de un análisis logístico multinivel aplicado a PISA-2006. Revista de Educación, núm. extr., 225-256.

Casquero, A., y Navarro, M. (2018). Determinantes del abandono escolar temprano en España, un análisis por género. Revista de Educación, núm. extr., 191-213.

Escudero Muñoz, J. M. (2005). Fracaso escolar, exclusión educativa: ¿De qué se excluye y cómo? Profesorado. Revista de Currículum y Formación del Profesorado, 1(1), 1-23. Disponible en: https://bit.ly/2SL8qeG

Escudero Muñoz, J. M.; González González, Ma . T., y Martínez Domínguez, B. (2009). El fracaso escolar como exclusión educativa: comprensión, políticas y prácticas. Revista Iberoamericana de Educación, 50, 41-64. Disponible en: https://bit.ly/2GJ15vl

Estrada de Madariaga, Ma del M. (2017). Estudio descriptivo sobre el abandono escolar temprano e influjo de variables personales y socioculturales en la Ciudad Autónoma de Melilla (Tesis Doctoral). Universidad de Granada, Granada.

Eurostat (2019, junio). Early leavers from education and training. Eurostat: Oficina Europea de Estadística. Disponible en: https://bit.ly/2MYlD4q

Fernández Enguita, M., Mena Martínez, L., y Riviere Gómez, J. (2010). Fracaso y abandono escolar en España. Colección Estudios Sociales. no 29. Barcelona. Fundación "La Caixa".

Ferrer Esteban, G., Castel Baldellou, J.L., y Ferrerán Ferrer, J. (2006). Las desigualdades del sistema educativo a través del estudio PISA 2003. Revista de Educación, núm. extr., 399- 428. Disponible en: https://bit.ly/2C9YhCz

Flick, U. (2014). La gestión de la calidad en investigación cualitativa. Madrid: Morata.

Gamoran, A. (2001). American schooling and educational inequality: A forecast for the 21st century. Sociology of Education. Extra Issue, 135-153. 
Hill Collins, P. (1990). Black Feminist Thought. Knowledge, consciousness, and the politics of empowerment. London: Routledge.

Hill Collins, P. (2009). Another kind of Public Education. Race, Schools, the Media and Democratic Possibilities. Boston: Beacon.

Hill Collins, P., y Bilge, S. (2016). Intersectionality. Malden, MA: Polity Press.

Jorganes, J. (2007). Rincón y Cortado van a la escuela. En J. Jorganes (2007), Algunas propuestas para la escuela del nuevo siglo (pp. 49-61). Madrid: MEC. FIES. Octaedro.

Finn, J. D., \& Rock, D.A. (1997). Academic Success Among Students at Risk for School Failure. Journal of Applied Psychology, 82(2), 221-234. Disponible en: https://bit.ly/2vlxBdJ

Lizasoain, L., Joaristi, L., Lukas, J. F., y Santiago, K. (2007). Efectos contextuales del nivel socioeconómico sobre el rendimiento académico en la educación secundaria obligatoria en la Comunidad Autónoma Vasca (España). Estudio diferencial del nivel socioeconómico familiar y el del centro escolar. EPAA/AAPE, 15, 1-37. Disponible en: http://www.redalyc.org/pdf/2750/275020546008.pdf

Marchesi, A. (2000). Un sistema de indicadores de desigualdad educativa. Revista Iberoamericana de Educación, 23, 135-163.

Marchesi, Á. (2003). El fracaso escolar en España. Madrid: Fundación Alternativas/ Documento de Trabajo 1/2003. Disponible en: https://bit.ly/2IwasKk

Mena Martínez, L., Fernández Enguita, M., y Riviére Gómez, J. (2010). Desenganchados de la educación: procesos, experiencias, motivaciones y estrategias del abandono y del fracaso escolar. Revista de Educación, núm. extr., 119-145.

Ministerio de Educación, Cultura y Deporte (2015). Plan para la reducción del abandono educativo temprano. Ministerio de Educación, Cultura y Deporte. Dirección General de Formación Profesional. Subdirección General de Aprendizaje a lo Largo de la Vida. Disponible en: https://bit.ly/2z81UsG

Ministerio de Educación y Formación Profesional (2017a). Sistema Estatal de Indicadores de la Educación 2017. Ministerio de Educación, Cultura y Deporte. Instituto Nacional de Evaluación Educativa. Subdirección General de Estadística y Estudios. Disponible en: https://bit.ly/2GLAWdJ

Ministerio de Educación y Formación Profesional (2017b). Datos y Cifras. Curso escolar 2017/2018. Ministerio de Educación y Formación Profesional. Secretaría General Técnica. Subdirección General de Estadística y Estudios. Disponible en: https://bit.ly/2EeR2XH

Ministerio de Educación y Formación Profesional (2018). Datos y Cifras. Curso escolar 2018/2019. Ministerio de Educación y Formación Profesional. Secretaría General Técnica. Subdirección General de Estadística y Estudios. Disponible en: https://bit.ly/2VCv6jg

Ministerio de Empleo y Seguridad Social (2018). Programa Nacional de Reformas. Reino de España 2018. Ministerio de Empleo y Seguridad Social. Subsecretaría de Empleo y Seguridad Social. Disponible en: https://bit.ly/2LErEPy

Ministerio de Educación y Formación Profesional (2019, 14 de febrero). Nivel de Formación, Formación Permanente y Abandono: Explotación de las variables educativas de la Encuesta de Población Activa.

Ministerio de Educación y Formación Profesional. Disponible en: https://bit.ly/2RrKAmW

Mora, A. J. (2010). Determinantes del abandono escolar en Cataluña, más allá del nivel socioeconómico de las familias. Revista de Educación, núm. extr., 171-190.

Moriña Díez, A. (2007). La exclusión social: análisis y propuestas para su prevención. Madrid: Fundación Alternativas. Disponible en: https://bit.ly/2IwasKk

OECD (2017). Education at a Glance 2017. OECD Indicators. OECD Publishing: Paris. doi: http://dx.doi.org/10.1787/eag-2017-en

OREAL/UNESCO Santiago (2013). Situación educativa de América Latina y el Caribe: Hacia la educación de calidad para todos al 2015. AECID/UNESCO. Disponible en: https://bit.ly/1evFNqD 
Page, B. (2011, 23 de marzo). Estudiantes en riesgo, ¿¿de qué? Educación Democrática-EUDEC. Disponible en: https://bit.ly/2Hlv2RH

Parrilla Latas, Á., Gallego Vega, C., y Moriña Díez, A. (2010). El complicado tránsito a la vida activa de jóvenes en riesgo de exclusión: una perspectiva biográfica. Revista de Educación, 351, 211-233.

Robinson, K. (2012). El elemento. Descubrir tu pasión lo cambia todo. Barcelona: Debolsillo.

Rumberger, R. W. (2001). Why students drop out of school and what can be done. Conference on Dropouts in America: How severe is the problem? What do we know about intervention and prevention? Civil Rights Project, California: UCLA. Disponible en: https://bit.ly/2G4NVWQ

Rumberger, R. W., y Ah Lin, S. (2008). Why students drop out of school: A review of 25 years of research. California Dropout Research Project Report \#15. Santa Barbara: University of California. Disponible en: https://bit.ly/2A9xR4O

Schraad-Tischler, D., Schiller, Ch., Matthias Heller, S., \& Siemer, N. (2017). Social Justice Index 2017 Social Inclusion Monitor Europe. Germay: Bertelsmann Stiftung.

Serrano, L., Soler, A., y Hernández, L. (2013). El abandono educativo temprano: análisis del caso español. Valencia: Ivie/Instituto Valenciano de Investigaciones Económicas.

Suárez, J. (2018) (ed.). Políticas para promover la culminación de la educación media en América Latina y el Caribe. Lecciones desde México y Chile. CAF/Banco de Desarrollo de América Latina. Disponible en: https://bit.ly/2Svi3OS

Susinos Rada, T., y Calvo Salvador, A. (2005-2006). "Yo no valgo para estudiar...". Un análisis crítico de la narración de las experiencias de exclusión social. Contextos educativos: Revista de educación, 8-9, 87-108.

Susinos Rada, T., y Parrilla Latas, Á. (2008). Dar la voz en la investigación inclusiva. Debates sobre inclusión y exclusión desde un enfoque biográfico-narrativo. REICE, 6(2), pp. 157-171. Disponible en: https://bit.ly/2LAOlaF

Torres Santomé. J. (2017). Políticas educativas y construcción de personalidades neoliberales y neocolonialistas. Madrid: Morata.

UNESCO (2012). International Standard Classification of Education. ISCED 2011. Ref: UIS/2012/INS/10/RE. Canada: UNESCO-UIS. Disponible en: https://bit.ly/2rFmZVK

Vázquez Recio, R. (2018) (Coord.). Hacia una literacidad del fracaso escolary del abandono temprano desde las voces de adolescentes y jóvenes: Resistencias, «cicatrices» y destinos. Cádiz. Editorial UCA.

Vázquez Recio, R. (2015). La organización de los tiempos de la infancia: entre lo institucionalizado y lo vivencial. Organización y gestión educativa, 6, 26-30.

Witte, Kr., De Cabus, S., Thyssen, G., Groot, W., \& Brink, H.M. van den (2013). A Critical Review of the Literature on School Dropout. Educational Research Review, 10, 13-28. doi: https://doi.org/10.1016/j.edurev.2013.05.002 


\section{Información sobre las autoras}

Autora: Rosa Vázquez Recio

Institución: Universidad de Cádiz

Email: rmaria.vazquez@uca.es

ORCID: https://orcid.org/0000-0001-6595-177X

Autora: Mónica López Gil

Institución: Universidad de Cádiz

Email: monica.maria@uca.es

ORCID: https://orcid.org/0000-0002-5412-2244

Autora: Guadalupe Calvo-García

Institución: Universidad de Cádiz

Email: guadalupe.calvo@uca.es

ORCID: https://orcid.org/0000-0001-6390-6561 


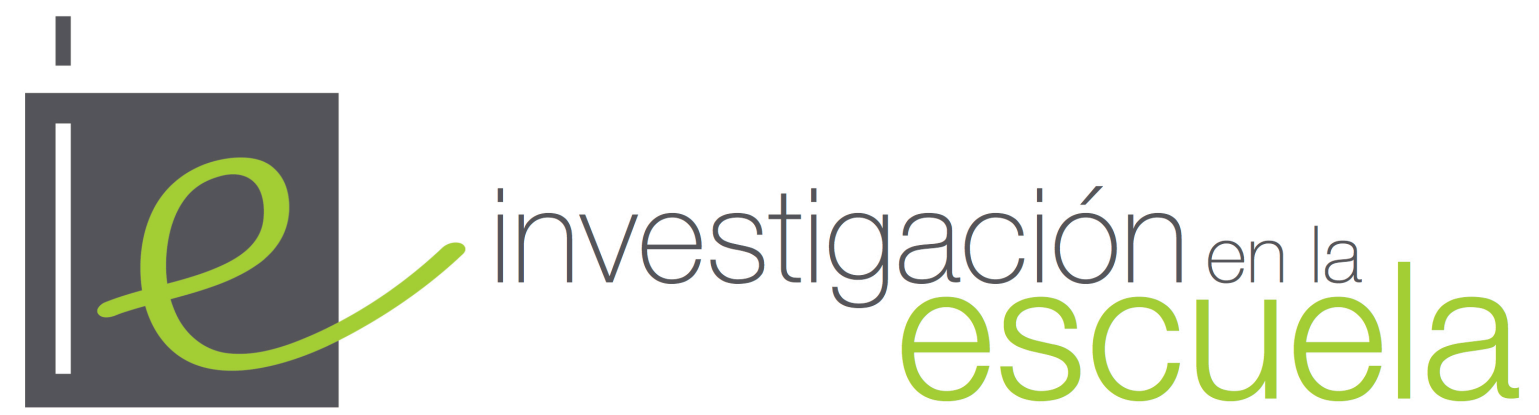

Revista académica evaluada por pares y de acceso abierto

Número 98

31 de julio de 2019

ISSN 2443-9991

\section{cc) (i) (2)}

Esta obra está bajo una licencia Creative Commons. Los/as lectores/as pueden compartir, copiar y redistribuir el material en cualquier medio o formato, así como adaptar, remezclar, transformar y construir a partir del material para cualquier propósito, incluso comercialmente. Para ello, deben de hacerlo bajo los siguientes términos: dando crédito de forma adecuada, brindando un enlace a la licencia e indicando si se han realizado cambios. Si se remezcla, transforma o crea a partir del material, debe distribuir su contribución bajo la misma licencia del original.

Más detalles de la licencia de CreativeCommons se encuentran en https://creativecommons.org/licenses/by-sa/4.0/deed.es

Cualquier otro uso debe ser aprobado en conjunto por el autor/es, o Investigación en la Escuela.

Uิ?

Revista Editada por la Universidad de Sevilla. https://editorial.us.es/es/revistainvestigacion-en-la-escuela

Por errores y sugerencias contacte a secretaria@investigacionenlaescuela.es

La revista Investigación en la Escuela desde su origen en 1987 hasta su nº 87 (2015) fue editada por Díada Editora. 
